\title{
Infrared interferometric observations of T Tauri stars
}

Rachel L. Akeson, David Ciardi, Gerard Theodore van Belle

Rachel L. Akeson, David Ciardi, Gerard Theodore van Belle, "Infrared interferometric observations of T Tauri stars," Proc. SPIE 4838, Interferometry for Optical Astronomy II, (21 February 2003); doi: 10.1117/12.457016

SPIE Event: Astronomical Telescopes and Instrumentation, 2002, Waikoloa, Hawai'i, United States 


\title{
Infrared interferometric observations of $\mathbf{T}$ Tauri stars
}

\author{
Rachel Akeson ${ }^{a}$, David Ciardi ${ }^{b}$ and Gerard van Belle ${ }^{c}$ \\ ${ }^{a}$ Interferometry Science Center, Caltech, Pasadena, CA, USA \\ ${ }^{b}$ Univ. of Florida, Gainesville, FL, USA \\ ${ }^{c}$ Jet Propulsion Laboratory, Caltech, CA, USA
}

\begin{abstract}
We present observations of several T Tauri stars using long baseline infrared interferometry from the Palomar Testbed Interferometer. The target sources, T Tau N, SU Aur, RY Tau and DR Tau, are all known to be surrounded by dusty circumstellar disks. The observations directly trace the inner regions $(<1 \mathrm{AU})$ of the disk and can be used to constrain the physical properties of this material. For three of the sources observed, the size scale of the infrared emission is tenths of $\mathrm{AU}$, which is considerably larger than predicted by flat disk models. We discuss the implications of these results for models of circumstellar material, in particular the recent theoretical work suggesting the presence of an extended vertical wall at the inner edge of the disk.
\end{abstract}

Keywords: Circumstellar material, young stellar objects, infrared interferometry

\section{INTRODUCTION}

Observational evidence for circumstellar material around most young stellar objects (YSOs) includes infrared emission in excess of the stellar photosphere, broad forbidden line profiles, and emission at millimeter wavelengths. Although the dust column density is inferred to be quite high, the sources are often optically visible, implying a geometrically flat distribution of the material. A disk morphology is also predicted by star formation theories as a consequence of conservation of angular momentum. These disks not only provide a conduit for material to accrete onto the central star, but are also a reservoir of material from which a potential planetary system might form.

Characterizing the physical properties of the inner disk is important for theories of hydrodynamic disk winds and for understanding the initial conditions of planet formation. The structure of T Tauri (YSO's with stellar mass up to a few $\mathrm{M}_{\odot}$ ) circumstellar disks has been studied using spectral energy distributions (SED's), spectral line profiles and imaging at infrared and (sub)-millimeter wavelengths. The infrared to millimeter wavelength continuum emission from the disk arises from dust emission. The millimeter emission from several $\mathrm{T}$ Tauri sources has been resolved (see review in Ref. 1). These observations are sensitive to emission from cooler dust and provide spatial information on size scales of several tens of AU. The disk physical properties on much smaller scales ( $<$ few AU) are generally inferred through examination of the spectral line shapes and modeling of the SED. Infrared interferometry provides a method to directly observe the inner disk.

\section{DATA}

Infrared interferometry data were taken at the Palomar Testbed Interferometer (PTI), which is described in detail in Ref. 2. PTI is a long-baseline, direct detection interferometer which utilizes active fringe tracking in the infrared. It was developed as a testbed for the Keck Interferometer, but is also used extensively for scientific observations. Data were obtained in the $\mathrm{K}$ band $(2.2 \mu \mathrm{m})$ in both the NS (110 meter) and NW (85 meter) baselines. The T Tau and SU Aur observations are described in Ref. 3. Observations of RY Tau and DR Tau were obtained between September 2001 and February 2002 and are described in Ref. 4.

Further author information: Send correspondence to R.A. E-mail:rla@ipac.caltech.edu 
The data were calibrated using the standard PTI method. ${ }^{5}$ Briefly, a synthetic wide-band channel is formed from five spectrometer channels $(\lambda=2.0-2.4)$. The system visibility, the response of the interferometer to an unresolved object, is measured using calibrator stars. Calibrator star sizes were estimated using a blackbody fit to photometric data from the literature and were internally consistent. Calibrators were chosen for their proximity to the source and for small angular size, minimizing systematic errors in deriving the system visibility. All calibrators used here have angular diameters $<0.8$ milliarcsec (mas) and were assigned uncertainties of 0.1 mas. The calibrated data are presented in normalized squared visibility $\left(\mathrm{V}^{2}=1\right.$ for an unresolved source). The calibrated visibility uncertainties are a combination of the calibrator size uncertainty and the internal scatter in the data. As DR Tau is near the sensitivity limit for PTI, the wide-band data are used rather than the synthetic wide-band (spectral channel) data.

\section{SOURCES}

All four sources are located in the Taurus-Aurigae molecular cloud (distance $\sim 140 \mathrm{pc}$ ) and are well studied T Tauri objects. Stellar properties are given in Table 1. We note that there are large variations in the literature for the total luminosity for some of the sources. For consistency, we have used the values from Ref. 6 for spectral type, effective temperature and luminosity.

\begin{tabular}{lclllll}
\hline Source & Sp Type & $\mathrm{T}_{\text {eff }}(K)$ & $\mathrm{L}_{\star}\left(L_{\odot}\right)$ & $\mathrm{A}_{V}$ & $\dot{M}\left(M_{\odot} / \mathrm{yr}\right)$ & Ref. \\
\hline T Tau N & K0 & 5250 & 15.5 & 1.4 & $4 \times 10^{-8}$ & 6,7 \\
SU Aur & G2 & 5860 & 12.9 & 0.9 & $1 \times 10^{-8}$ & 6,7 \\
RY Tau & K1 & 5080 & 16.7 & 0.55 & $2.5 \times 10^{-8}$ & 6,8 \\
DR Tau & K7 & 4060 & 3.0 & $1.2-3.2$ & $3 \times 10^{-7}-8 \times 10^{-6}$ & $6-8$ \\
\hline
\end{tabular}

Table 1. Stellar parameters for the observed sources.

Notes on individual sources:

- The T Tau system comprises the optically visible star T Tau N and its infrared companion T Tau S, which is itself a binary. ${ }^{9}$ The PTI observations are of T Tau N, the component which dominates the millimeter emission. ${ }^{10}$ The incoherent contribution from $\mathrm{T}$ Tau $\mathrm{S}$ has been removed from the presented visibilities using flux ratios measured by T. Beck.

- SU Aur has an SED similar to that of T Tau N, although Ref. 11 classified SU Aur separately from other $\mathrm{T}$ Tauri's due to its high luminosity and broad absorption lines.

- RY Tau is associated with a reflection nebulosity and has molecular line emission consistent with a Keplerian disk. ${ }^{12}$

- DR Tau is one of the most heavily veiled T Tauri stars and is highly variable in the optical. ${ }^{7}$ As the stellar photosphere is not visible even in the optical, the estimates for extinction and accretion rate in the literature have a wide range (Table 1).

\subsection{Spectral energy distributions}

Optical and infrared photometry for each source were taken from the literature compilation of Ref. 6 and used to determine the contribution of the stellar photosphere in the K band (Figure 1). For all sources except DR Tau, the stellar photosphere was fit using the de-reddened optical photometry and the blackbody temperature given in Table 1. DR Tau is so heavily veiled that the optical flux is not dominated by the stellar photosphere. For this source, a luminosity of $1.7 \mathrm{~L}_{\odot}^{8}$ was used to set the flux level using an extinction of 2.0 magnitudes. 

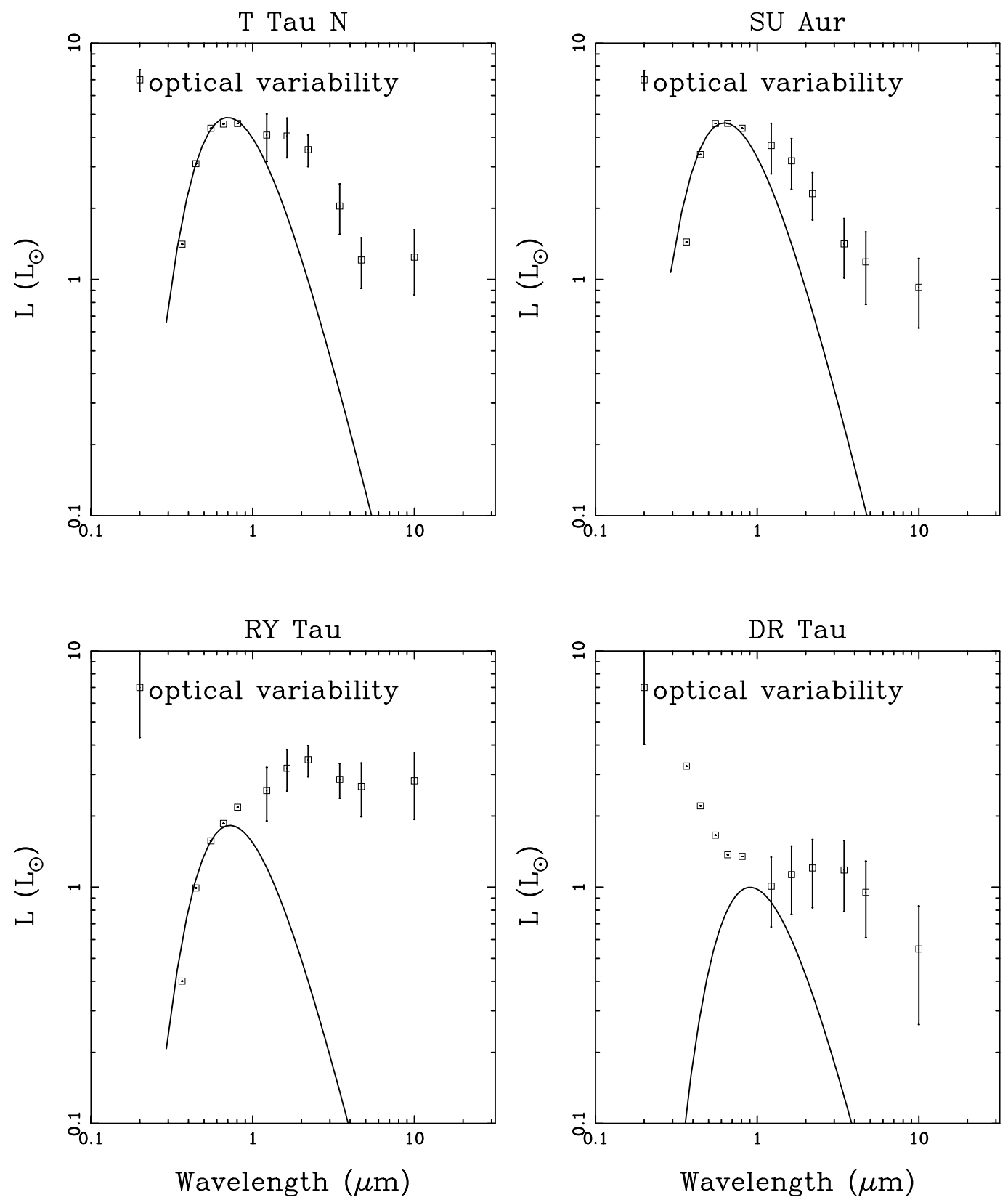

Figure 1. De-reddened spectral energy distribution for each source (points) and the estimated photospheric contribution (line). Note that the error bars plotted for the infrared photometry include source variability, while for the optical photometry the variability is shown separately.

\section{SIZE SCALE OF K BAND EMISSION}

The photometric contribution for each source was removed from the visibilities using the fits shown in Figure 1. T Tau N, SU Aur and RY Tau all have visibility less than 1, while the data from DR Tau is consistent with an unresolved source. At the distance to these sources $(\sim 140 \mathrm{pc})$, a star with a stellar radius of a few $\mathrm{R}_{\odot}$ (typical for $\mathrm{T}$ Tauris) subtends an angular diameter less than 0.1 mas, which is not resolvable at PTI.

A detailed discussion of the scenarios that could produce a visibility of less than 1 is contained in Ref. 3 . Briefly, these include binary companions, other sources within the field of view and a resolved source of emission. $\mathrm{T}$ Tau $\mathrm{N}$ is known to have a companion which contributes to the measured visibilities, and its visibilities have 
been calibrated for this effect as described in Ref. 13. Given the lack of evidence for companions for the other sources and the lack of time variation in the visibilities, we attribute the resolved visibility as arising from a combination of a unresolved photosphere and a circumstellar disk component.

We fit a Gaussian brightness distribution to the data as an estimate of the size scale of the $\mathrm{K}$ band emission from the disk. The errors listed in the table are from the measurement and calibration uncertainties. There is an additional error component due to the uncertainty in the photosphere-to-disk flux ratio. This uncertainty is difficult to estimate given that the variability is attributed to variable stellar extinction and changes in the accretion properties. ${ }^{14}$

\begin{tabular}{llllll}
\hline Source & $\begin{array}{l}\text { FWHM } \\
\text { mas }\end{array}$ & $\begin{array}{l}\sigma_{\text {data }} \\
\text { mas }\end{array}$ & $\begin{array}{l}\text { FWHM } \\
\text { AU }\end{array}$ & $\begin{array}{l}\text { Position } \\
\text { angle }(\text { deg })\end{array}$ & $\begin{array}{l}\text { Inclination } \\
\text { angle }(\text { deg })\end{array}$ \\
\hline T Tau N & 1.54 & 0.06 & 0.21 & $132 \pm 20$ & $29 \pm 15$ \\
SU Aur & 1.05 & 0.08 & 0.15 & $127 \pm 10$ & $62 \pm 8$ \\
RY Tau & 1.96 & 0.05 & 0.27 & $62 \pm 10$ & $30 \pm 3$ \\
DR Tau & unres & 0.8 & unres & - & - \\
\hline
\end{tabular}

Table 2. Results of Gaussian fits. The uncertainty given $\left(\sigma_{\text {data }}\right)$ is for the calibrated visibilities only and does not include the error in estimating the stellar/disk ratio.

As discussed in Ref. 3, the size scale of the $\mathrm{K}$ band emission indicated by the measured visibilities for $\mathrm{T}$ Tau N, SU Aur and RY Tau is considerably larger than the size predicted by flat accretion disk models with parameters set by fitting the SED.

\subsection{Ring Model}

Recent observations and theoretical work on the issue of inner disk structure have been done on Herbig Ae/Be stars (hereafter HAeBe), the higher mass analogs of T Tauri's. Ref. 15 observed 15 HAeBe's with the IOTA interferometer and found characteristic sizes larger than expected. Their measured visibilities were generally more consistent with spherical envelopes or thin shells than with disk models. Ref. 16 used aperture masking to image LkH $\alpha 101$ and found an inner edge for the infrared emission at $3.4 \mathrm{AU}$, inferring that this position is set by dust sublimation. Recent theoretical work proposes that a thick inner wall at the dust sublimation radius would solve the discrepancy between near-infrared observations and previous models. ${ }^{17,18}$ Optically thin material may be present within this radius.

To test this hypothesis, we fit a simple ring model to each source (Table 3 ). The ring has a fixed width of $10 \%$. We compare the fit radius to the radius at which the dust temperature is $1800 \mathrm{~K}$, chosen to represent the dust sublimation temperature. The dust radius is calculated using the stellar luminosity given in Table 1. As shown in Figure 2, there is a correlation between these two quantities. We note that the model in Refs.17,18 may only be valid for sources with low $\left(10^{-8} \mathrm{M}_{\odot} / \mathrm{yr}\right)$ accretion rates and that this assumption is almost certainly not valid for DR Tau.

\begin{tabular}{llllll}
\hline Source & Fit radius & $\sigma$ & Fit radius & $\mathrm{R}_{\star}$ & Fit radius $/ \mathrm{R}_{\star}$ \\
& mas & mas & $\mathrm{AU}$ & $\mathrm{R}_{\odot}$ & \\
\hline T Tau N & 0.89 & 0.032 & 0.12 & 4.7 & 5.5 \\
SU Aur & 0.73 & 0.033 & 0.10 & 3.4 & 6.3 \\
RY Tau & 1.12 & 0.025 & 0.16 & 5.2 & 6.6 \\
DR Tau & unres & 0.4 & unres & 3.5 & $<2.4$ \\
\hline
\end{tabular}

Table 3. Results of ring fits. The ring has a fixed width of $10 \%$. As with the Gaussian fits, the uncertainty given is for the calibrated visibilities only and does not include the error in estimating the stellar/disk ratio. 


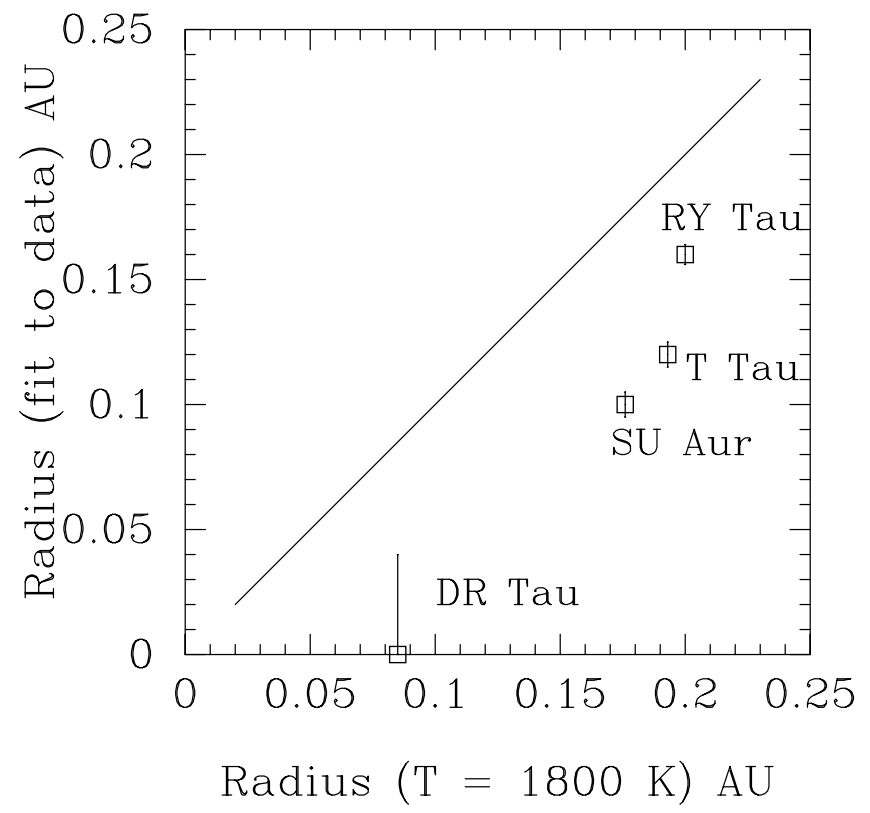

Figure 2. Comparison of the radius at which the dust temperature is $1800 \mathrm{~K}$ and the radius of a ring with $10 \%$ width fit to the data. Sources for which these two radii were equal would lie along the diagonal line.

\section{DISCUSSION}

Three of the four $\mathrm{T}$ Tauri sources observed are resolved in the infrared on milliarcsec scales, while the fourth is unresolved. These results are consistent with the predictions of recent theoretical work. ${ }^{17,18}$ Interestingly, DR Tau, which is unresolved, has an accretion rate at least an order of magnitude higher than the other sources and the lowest effective temperature, both of which would tend to decrease the size of the disk inner radius. The measured ring radius is consistently smaller than the size predicted for a dust temperature of $1800 \mathrm{~K}$. This could be due to an underestimation of the dust sublimation temperature or an overestimation of the stellar luminosity. We note that other estimates in the literature for the stellar luminosities of these sources tend to be lower than those used here.

In Table 3, we estimate the stellar radius using the luminosity and effective temperature values given in Table 1. We can then calculate the measured size (using the ring fit) in relation to the stellar radius. For the resolved sources, this value is roughly $5-6 \mathrm{R}_{\star}$. In theoretical models of accretion disks including stellar magnetic fields the inner disk is truncated near the radius where the disk and stellar rotational angular velocities are equal. ${ }^{19}$ For accretion rates of $\sim 10^{-8} \mathrm{M}_{\odot} / \mathrm{yr}$, this truncation radius is $\sim 5 \mathrm{R}_{\star},{ }^{20}$ roughly the value calculated for the three resolved sources, which all have accretion rates within a factor of a few of $10^{-8} \mathrm{M}_{\odot} / \mathrm{yr}$.

Due to sensitivity constraints at PTI, the sources presented here are not representative of an average T Tauri object, but are brighter and more luminous. Further observations of a wider range of $\mathrm{T}$ Tauri objects are needed to characterize the inner disk properties as a function stellar properties, such as age, effective temperature and accretion rate. The generation of interferometers currently under development, including the Keck Interferometer, the Very Large Telescope Interferometer and the CHARA array, will be well suited for these studies.

\section{ACKNOWLEDGMENTS}

This work was performed at the Interferometry Science Center and the Jet Propulsion Laboratory, California Institute of Technology, under a contract with the National Aeronautics and Space Adminstration. Data were 
obtained at the Palomar Observatory using the NASA Palomar Testbed Interferometer. Science operations with PTI are possible through the efforts of the PTI Collaboration(http://huey.jpl.nasa.gov/palomar/

ptimembers.html). This work has made use of software produced by the Interferometry Science Center at the California Institute of Technology. We are grateful to T. Beck for providing the T Tau flux ratio.

\section{REFERENCES}

1. D. J. Wilner and O. P. Lay, "Subarcsecond Millimeter and Submillimeter Observations of Circumstellar Disks," Protostars and Planets IV, pp. 509+, May 2000.

2. M. M. Colavita, J. K. Wallace, B. E. Hines, Y. Gursel, F. Malbet, D. L. Palmer, X. P. Pan, M. Shao, J. W. Yu, A. F. Boden, P. J. Dumont, J. Gubler, C. D. Koresko, S. R. Kulkarni, B. F. Lane, D. W. Mobley, and G. T. van Belle, "The Palomar Testbed Interferometer," Astrophysical Journal 510, pp. 505-521, Jan. 1999.

3. R. L. Akeson, D. R. Ciardi, G. T. van Belle, and M. J. Creech-Eakman, "Constraints on Circumstellar Disk Parameters from Multiwavelength Observations: T Tauri and SU Aurigae," Astrophysical Journal 566, pp. 1124-1131, Feb. 2002.

4. R. L. Akeson, D. R. Ciardi, and G. T. van Belle. in preparation.

5. A. F. Boden, M. M. Colavita, G. T. van Belle, and M. Shao, "Visibility calibrations with the Palomar Testbed Interferometer," in Proc. SPIE Vol. 3350, p. 872-880, Astronomical Interferometry, Robert D. Reasenberg; Ed., 3350, pp. 872-880, July 1998.

6. S. J. Kenyon and L. Hartmann, "Pre-Main-Sequence Evolution in the Taurus-Auriga Molecular Cloud," Astrophysical Journals 101, pp. 117+, Nov. 1995.

7. E. Gullbring, N. Calvet, J. Muzerolle, and L. Hartmann, "The Structure and Emission of the Accretion Shock in T Tauri Stars. II. The Ultraviolet-Continuum Emission," Astrophysical Journal 544, pp. 927-932, Dec. 2000.

8. P. Hartigan, S. Edwards, and L. Ghandour, "Disk Accretion and Mass Loss from Young Stars," Astrophysical Journal 452, pp. 736+, Oct. 1995.

9. C. D. Koresko, "A Third Star in the T Tauri System," Astrophysical Journal 531, pp. L147-L149, Mar. 2000.

10. R. L. Akeson, D. W. Koerner, and E. L. N. Jensen, "A Circumstellar Dust Disk around T Tauri N: Subarcsecond Imaging at lambda $=3$ Millimeters," Astrophysical Journal 505, pp. 358-362, Sept. 1998.

11. G. H. Herbig and K. R. Bell, Catalog of emission line stars of the orion population : $3: 1988$, Lick Observatory Bulletin, Santa Cruz: Lick Observatory, —c1988, 1988.

12. D. W. Koerner and A. I. Sargent, "Imaging the Small-Scale Circumstellar Gas Around T Tauri Stars," Astronomical Journal 109, pp. 2138+, May 1995.

13. R. L. Akeson, D. R. Ciardi, G. T. van Belle, M. J. Creech-Eakman, and E. A. Lada, "Infrared Interferometric Observations of Young Stellar Objects," Astrophysical Journal 543, pp. 313-317, Nov. 2000.

14. M. F. Skrutskie, M. R. Meyer, D. Whalen, and C. Hamilton, "Near-Infrared Photometric Monitoring of Young Stellar Objects," Astronomical Journal 112, pp. 2168+, Nov. 1996.

15. R. Millan-Gabet, F. P. Schloerb, and W. A. Traub, "Spatially Resolved Circumstellar Structure of Herbig AE/BE Stars in the Near-Infrared," Astrophysical Journal 546, pp. 358-381, Jan. 2001.

16. P. G. Tuthill, J. D. Monnier, and W. C. Danchi, "A dusty torus around the luminous young star LkH\&alpha;101," Nature 409, pp. 1012-1014, Feb. 2001.

17. A. Natta, T. Prusti, R. Neri, D. Wooden, V. P. Grinin, and V. Mannings, "A reconsideration of disk properties in Herbig Ae stars," Astronomy and Astrophysics 371, pp. 186-197, May 2001.

18. C. P. Dullemond, C. Dominik, and A. Natta, "Passive Irradiated Circumstellar Disks with an Inner Hole," Astrophysical Journal 560, pp. 957-969, Oct. 2001.

19. F. Shu, J. Najita, E. Ostriker, F. Wilkin, S. Ruden, and S. Lizano, "Magnetocentrifugally driven flows from young stars and disks. 1: A generalized model," Astrophysical Journal 429, pp. 781-796, July 1994.

20. L. Hartmann, "On Disk Braking of T Tauri Rotation," Astrophysical Journal Letters 566, pp. L29-L32, Feb. 2002. 